\title{
Operational Status Evaluation for Electric Vehicle Chargers based on Layered Radar Map Method
}

\author{
Tao Jiang ${ }^{1, a}$, Weiyong $\mathrm{Yu}^{1, \mathrm{~b}}$, Jian $\mathrm{Hu}^{1, \mathrm{c}}$, Zhen Luo ${ }^{1, \mathrm{~d}}$, Yushuang $\mathrm{Li}^{2, \mathrm{e}}$ and Fan \\ $Y e^{2, f}$ \\ ${ }^{1}$ Yuhang Power Supply Company of State Grid Zhejiang Hangzhou Electric Power Company, \\ Hangzhou 311100, China; \\ ${ }^{2}$ State Key Laboratory of Power Transmission Equipment \& System Security and New Technology, \\ Chongqing University, Chongqing 400044, China \\ ª415397022@qq.com, b745328181@qq.com, '871965882@qq.com, d529737265@qq.com, \\ 4451592205@qq.com
}

\begin{abstract}
Keywords: electric vehicle changers; status evaluation; indices selection; factor analysis; radar map Abstract. For optimizing the maintenance strategy of electric vehicle (EV) changers, it's necessary to have a comprehensive evaluation for it by using its monitoring data. From the battery, electrical machinery transmission and charge-discharge process, 26 characteristic quantities are chosen as EV changers operation state indicators, the common factor indicators are extracted by using the factor analysis method and then the EV changers state indicators are classified by the independent common factors; the classified indicators are screened by using stepwise regression analysis, and the indicators that contribute little to the evaluation result are excluded, thereby the EV changers operation state comprehensive evaluation system are established. Combined hierarchical structure of EV changers evaluation and radar map method, the layered radar map method is proposed for EV changers comprehensive evaluation.
\end{abstract}

\section{Introduction}

Compared to conventional cars, electric vehicles have incomparable advantages in promoting energy conservation and emission reduction, reducing operating costs and generalizing new energy utilization [1]. With EV abundantly available, it will have influence on the security and stability of power gird, and also bring hardship to the repair and maintenance of EV chargers [2-4]. Monitoring and evaluating the operating state of EV chargers timely and accurately, can help deal with the fault and optimize the maintenance strategy of EV chargers.

The EV changers operation state comprehensive evaluation system based on visualization technology, not only can visualize the relationship among the indicators, but also guide the repair strategy graphically, which must solve the classification and the reduction of dimension of multiple indicators, and the graphical display of assessment results [5]. According to the battery, electrical machinery transmission and charge-discharge process, this paper chooses EV changers operation state indicators comprehensively and objectively. The EV changers state indicators are classified by using the factor analysis method, and the indicators that contribute little to the evaluation result are excluded by using stepwise regression analysis, thereby the EV changers operation state comprehensive evaluation system are established. The layered radar map method is proposed for EV changers comprehensive evaluation, making the different levels of EV changers multi-dimensional status indicators represent in the radar map visually.

\section{Selection of EV changers operation state indicators}

The electrical structure of the EV changers includes the power converter, storage battery and electrical machinery. According to the battery and electromotor state characteristic quantities in the running process, and the electrical characteristic quantities of the charge-discharge process, 26 indicators are chosen as EV changers operation state indicators, as shown in Table 1. 
Tab. 1 Operational status indices of electric vehicles

\begin{tabular}{cccc}
\hline No & Name & No & Name \\
\hline 1 & Charging voltage deviation & 14 & Battery termination voltage \\
2 & Charging frequency deviation & 15 & Discharge duration \\
3 & Charging voltage flicker & 16 & Battery output \\
4 & Charging voltage unbalance & 17 & Self-discharge rate \\
5 & Charging voltage fluctuation & 18 & Battery cycle life factor \\
6 & Charging power factor & 19 & Battery temperature matching coefficient \\
7 & Charging current harmonics & 20 & The initial SOC matching coefficient \\
8 & Energy loss & 21 & Battery voltage matching coefficient \\
9 & Discharge efficiency & 22 & Motor temperature \\
10 & Energy consumption per mile & 23 & MTBF \\
11 & Discharge depth & 24 & Maximum speed \\
12 & Charge and discharge times & 25 & Driven distance \\
13 & Battery capacity & 26 & Motor overload factor \\
\hline
\end{tabular}

The operation state of the battery, electrical machinery and other different modules can be obtained by using the state indicators, and the overall performance of EV changers can also be obtained through a comprehensive evaluation. Because of the correlations between the different modules and the different indicators in the same module of EV changers, it will have a strong impact on the evaluation result. So, there is a need to classify and screen the EV changers state indicators that given in Table 1.

\section{Classification and screening of EV changers operation state indicators based on factor analysis}

Assuming the number of samples EV changers operation state is $n$ and the number of indicators is $m=26$, where $V_{1}$ and $V_{2}$ represent reverse and appropriate indicators set respectively. The j-th indicators value of the i-th sample trends into,

$$
x_{i j}^{\prime}=\left\{\begin{array}{lr}
\max _{1 \leq i \leq n}\left\{x_{i j}^{\prime \prime}\right\}-x_{i j}^{\prime \prime} & , v_{j} \in \boldsymbol{V}_{1} \\
\max _{1 \leq i \leq n}\left\{\left|x_{i j}^{\prime \prime}-x_{j}^{\prime \prime}\right|\right\}-\left|x_{i j}^{\prime \prime}-x_{j}^{\prime \prime}\right|, v_{j} \in \boldsymbol{V}_{2}
\end{array}\right.
$$

Where $x_{i j}^{\prime \prime}$ and $x_{i j}$ is the indicators value before and after the trend, $x_{\mathrm{j}}^{\prime \prime}$ is the appropriate value of the $\mathrm{j}$-th indicators (appropriate indicators $x^{\prime \prime}{ }_{i j}$ closer to $x_{\mathrm{j}}^{\prime \prime}$ is better). Then calculating the standardized state indicators $x_{i j}$,

$$
x_{i j}=\frac{x_{i j}^{\prime}-\frac{1}{n} \sum_{i=1}^{n} x_{i j}^{\prime}}{\sqrt{\frac{1}{n-1} \sum_{i=1}^{n}\left(x_{i j}^{\prime}-\frac{1}{n} \sum_{i=1}^{n} x_{i j}^{\prime}\right)^{2}}}
$$

The common factor state indicators are extracted by using the factor analysis method to the sample data, which will contain multiple state indicators with close relationship. And then the EV changers state indicators are classified whereby it. 


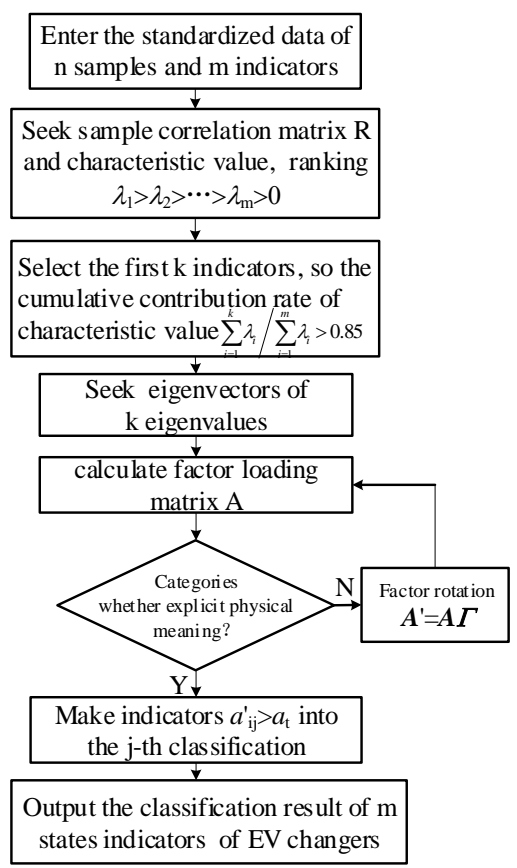

Fig. 1 Flow chart of state indicators classification

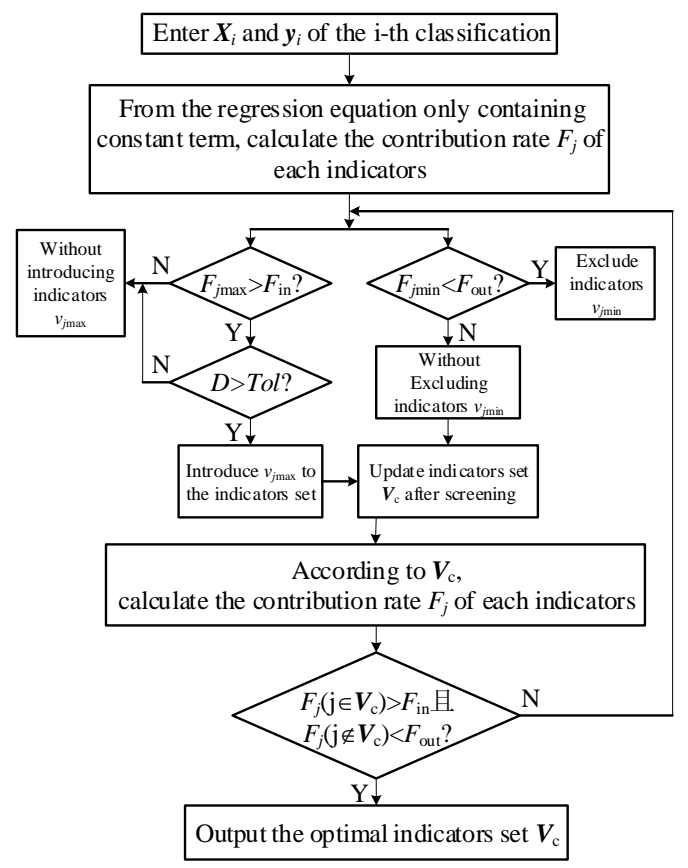

Fig. 2 Flow chart of status indices selection

Flow chart of state indicators classification by factor analysis is shown in Fig. 1. According to the real-time monitoring data of an EV changer, the coefficient matrix characteristic value related to the sample data can be obtained when state indicators data are standardized. And the $26 \mathrm{EV}$ changers operation state indicators come down to 5 types of $f_{1}$ to $f_{5}$, (common factors), including battery efficiency, battery lifetime, battery charge-discharge characteristic, battery cell consistency and electrical machinery operation state. Where battery efficiency contains 3 indicators of $V_{8}$ to $V_{10}$, battery lifetime contains 5 indicators of $V_{11}$ to $V_{12}$ and $V_{17}$ to $V_{18}$, battery charge-discharge contains 9 indicators of $V_{1}$ to $V_{7}$ and $V_{14}$ to $V_{15}$, battery cell consistency contains 3 indicators of $V_{19}$ to $V_{21}$, electrical machinery operation state contains 6 indicators of $V_{19}$, and $V_{22}$ to $V_{26}$.

Choosing a skimp number of indicators will increase the evaluation error of $\mathrm{EV}$ changers operation state, however, an excessive number of indicators will increase the complexity of models and reduces its practicability. Thus, it is necessary to screen the 5 subsets of classification indicator of $f_{1}$ to $f_{5}$ further.

Flow chart of state indicators selection by stepwise regression analysis is shown in Fig. 2. Calculating the contribution rate of the indicators $F_{\mathrm{j}}$, in accordance with the $\mathrm{i}$-th classification indicators set $V_{\mathrm{c}}$ after stepwise selections. If $F_{\text {jmax }}$ of the $\mathrm{j}_{\max }$-th indicator satisfies $F_{j \max }=\max \left(F_{j}\right)>F_{\text {in }}$ and acceptable level $D>T o l, V_{\mathrm{c}}$ introduces it, otherwise not. If $F_{\mathrm{jmin}}$ of the $\mathrm{j}_{\min }$-th indicator satisfies $F_{j \min }=\min \left(F_{j}\right)<F_{\text {out }}, V_{\mathrm{c}}$ excludes it, otherwise not. When all the contribution rate of the indicators in the $V_{\mathrm{c}}$ is greater than $F_{\text {out }}$, and outside the $V_{\mathrm{c}}$ is less than $F_{\text {in }}$, the Screening ends and the optimal indicators set can be outputted.

The EV changers operation state comprehensive evaluation system are established by the selection, classification and screening of EV changers operation state indicators, as shown in Fig. 3. The figure includes 3 layers of a target layer, a classification layer and an indicator layer. The target layer is divided into 5 categories, and refines the indicators under each category. For easy analysis, the indicators under different categories are renumbered. 


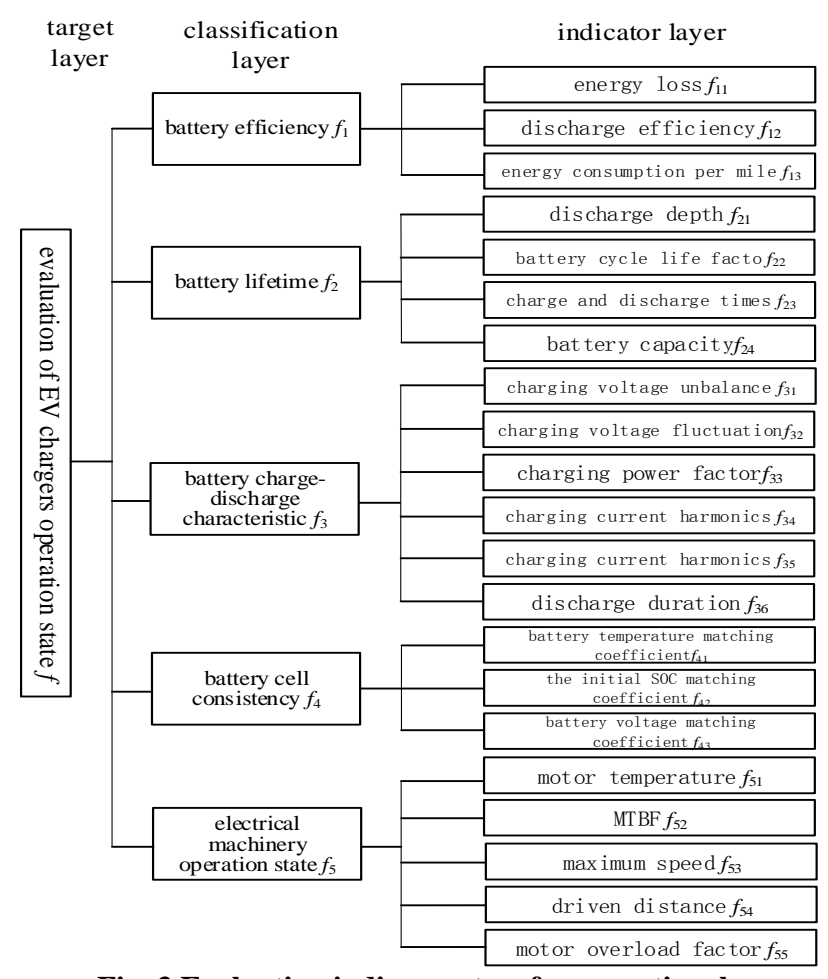

Fig. 3 Evaluation indices system for operational

\section{Comprehensive evaluation for EV chargers operation state based on layered radar map method}

The EV changers operation state comprehensive evaluation can be divided into three-layer analysis models by the method described above. Combined the hierarchy structure in Fig. 3 with the radar map method, the layered evaluation method of EV changers operation state can be achieved. Schematic diagram of comprehensive evaluation based on layered radar map is shown in Fig. 4. First, the radar map of each layer can be obtained using the sample data of the indicator layer, and got the radar map evaluation value of each classification through the image feature. And then according to the evaluation value, the evaluation result of the target layer of the EV changers operation state can be obtained. The assessment steps of layered radar map method are as follows.

1) Calculate the comprehensive weights of the indicator layer and classification layer combined with the improved subjective weighting method and objective entropy weight method.

2) Draw the first unit length line OA vertically up, taking the center as the starting point. Letting the first indicator weight convert to an angle value, draw the second unit length line $\mathrm{OB}$, and the rest lines are in the same way.

3) Draw the bisector of angle of each sector, taking the center as the starting point. Each normalized indicator is used as the length of the bisector of angle, and connecting the periphery points sequentially to get the radar map. Using the total area of polygon $\mathrm{S}$ and the perimeter $\mathrm{C}$, the quantitative evaluation value $\sqrt{S C}$ is obtained.

4) Repeat step 2) and 3) to get the radar map and its evaluation value of 5 categories.

5) According to the evaluation value of 5 categories, the radar map and comprehensive evaluation value of the EV changers operation state is obtained by step 2) and 3). And the comprehensive level of the sample waiting for the evaluation is determined by the different levels of standard samples. 


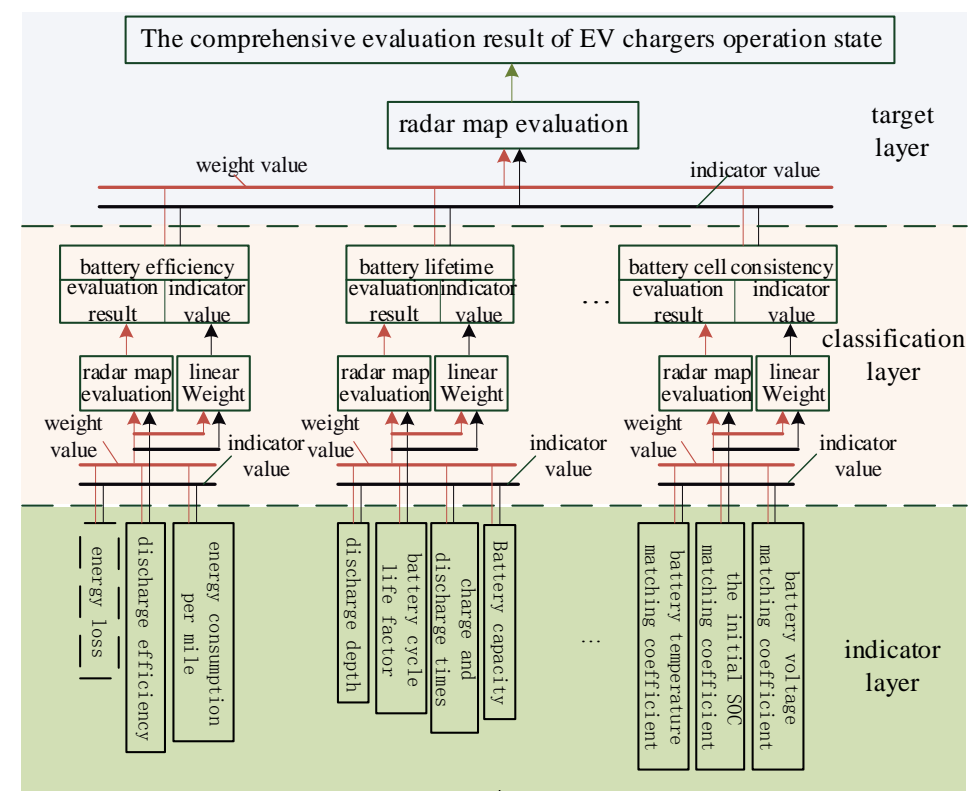

Fig. 4 Schematic diagram of comprehensive evaluation based on layered radar map

\section{Conclusions}

This paper divided the $26 \mathrm{EV}$ changers operation state indicators into five classifications, including battery efficiency, battery lifetime, battery charge-discharge characteristic, battery cell consistency and electrical machinery operation state by factor analysis. And the redundant indicators of each classification are screened by using stepwise regression analysis, thereby the practical EV changers operation state comprehensive evaluation system are established. Combined hierarchical structure of EV changers evaluation and radar map method, the comprehensive evaluation result of EV chargers operation state is obtained. The EV changers operation state comprehensive evaluation system proposed in the paper can reflect the real state of EV chargers better, and the evaluation result can provide technical reference for the maintenance of EV chargers.

\section{References}

[1] Wang Xifan, Shao Chengcheng, Wang Xiuli, et al. Survey of electric vehicle charging load and dispatch control strategies[J]. Proceedings of the CSEE, 2013, 33(1): 1-10.

[2] Yilmaz M, Krein P T. Review of battery charger topologies, charging power levels, and infrastructure for plug-in electric and hybrid vehicles[J]. IEEE Transactions on Power Electronics, 2013, 28(5): 2151-2169.

[3] Li Huiling, Bai Xiaomin. Impacts of electric vehicles charging on distribution grid [J]. Automation of Electric Power Systems, 2011, 35(17): 38-43.

[4] Zhou Niancheng, Xiong Xicong, Wang Qianggang. Simulation of charging load probability for connection of different electric vehicles to distribution network $[\mathrm{J}]$. Electric Power Automation Equipment, 2014, 32(2): 1-7.

[5] Wang Deqing, Wan Yongbo, Wang Xiang, et al. Improved radar chart based on principal component and its application in comprehensive evaluating [J]. Journal of Applied Statistics and Management, 2010, 29(5): 883-889 